Corrigendum

\title{
Corrigendum to "Association between Virulence Factors and Extended Spectrum Beta-Lactamase Producing Klebsiella pneumoniae Compared to Nonproducing Isolates"
}

\author{
Mustafa Muhammad Gharrah, ${ }^{1}$ Areej Mostafa El-Mahdy $\mathbb{D}^{1},{ }^{1,2}$ and Rasha Fathy Barwa $\mathbb{D}^{1}$ \\ ${ }^{1}$ Microbiology \& Immunology Department, Faculty of Pharmacy, Mansoura University, Mansoura 35516, Egypt \\ ${ }^{2}$ Department of Pharmaceutical Sciences, College of Pharmacy, Princess Norah Bint Abdulrahman University, \\ Riyadh 11671, Saudi Arabia \\ Correspondence should be addressed to Rasha Fathy Barwa; rasha2000@gmail.com
}

Received 11 December 2017; Accepted 14 December 2017; Published 8 January 2018

Copyright (c) 2018 Mustafa Muhammad Gharrah et al. This is an open access article distributed under the Creative Commons Attribution License, which permits unrestricted use, distribution, and reproduction in any medium, provided the original work is properly cited.

In the article titled "Association between Virulence Factors and Extended Spectrum Beta-Lactamase Producing Klebsiella pneumoniae Compared to Nonproducing Isolates" [1], there were errors, which should be corrected as follows:

(i) In the Results section, "a total of twenty-four different virulence profiles were observed among the tested isolates," should be "a total of twenty-three different virulence profiles were observed among the tested isolates."

(ii) In the Discussion section, "analysis of virulence factors combination has brought out 23 different virulence profiles including 2 to 8 virulence factors (Table 4)," should be "analysis of virulence factors combination has brought out 23 different virulence profiles including 2 to 9 virulence factors (Table 4)."

(iii) In the eighteenth row of Table 4, "Biofilm-serum resistant-haemagglutination-lipase- $\alpha$-hemolysis-BssSfimH-iss" should be corrected to "Biofilm-serum resistant-haemagglutination-hypermucoviscosity-lipase- $\alpha$-hemolysis-BssS-fimH-iss." The correct table is shown as follows: 
TABLE 4: Virulence gene profiles associated with ESBLs and non-ESBLs producing K. pneumoniae isolates.

\begin{tabular}{|c|c|c|}
\hline Virulence profiles & $\begin{array}{c}\text { ESBLs } \\
\text { producing } \\
\text { isolates, number } \\
(\%)\end{array}$ & $\begin{array}{c}\text { Non-ESBLs } \\
\text { producing } \\
\text { isolates, number } \\
(\%)\end{array}$ \\
\hline Biofilm-haemagglutination-BssS-fimH & $11(22 \%)$ & $12(24 \%)$ \\
\hline Biofilm-haemagglutination-hypermucoviscosity-BssS-fimH-iss-iucA & $1(2 \%)$ & $1(2 \%)$ \\
\hline Biofilm-haemagglutination-BssS-fimH-iss & $6(12 \%)$ & $1(2 \%)$ \\
\hline Biofilm-serum resistant-haemagglutination-BssS-fimH & $7(14 \%)$ & $1(2 \%)$ \\
\hline Biofilm-serum resistant-haemagglutination-BssS-fimH-iss & $14(28 \%)$ & $3(6 \%)$ \\
\hline Biofilm-BssS-fimH & $1(2 \%)$ & $1(2 \%)$ \\
\hline Biofilm-serum resistant-haemagglutination-lipase-BssS-fimH & $2(4 \%)$ & $0(0 \%)$ \\
\hline Biofilm-serum resistant-haemagglutination-BssS-fimH-iss-iucA & $3(6 \%)$ & $0(0 \%)$ \\
\hline Biofilm-serum resistant-haemagglutination-lipase- $\alpha$ hemolysis- $B s s S$-fim $H$ & $1(2 \%)$ & $0(0 \%)$ \\
\hline Biofilm-serum resistant-haemagglutination-BssS-fimH-iucA & $1(2 \%)$ & $0(0 \%)$ \\
\hline BssS-fimH & $1(2 \%)$ & $0(0 \%)$ \\
\hline $\begin{array}{l}\text { Biofilm-serum } \\
\text { resistant-haemagglutination-hypermucoviscosity-BssS-fimH-iss-iucA }\end{array}$ & $1(2 \%)$ & $0(0 \%)$ \\
\hline Biofilm-haemagglutination-gelatinase- $B s s S-f i m H$ & $1(2 \%)$ & $1(2 \%)$ \\
\hline Hypermucoviscosity-BssS-fimH & $0(0 \%)$ & $2(4 \%)$ \\
\hline Biofilm-haemagglutination-hypermucoviscosity-BssS-fimH & $0(0 \%)$ & $18(36 \%)$ \\
\hline Biofilm-haemagglutination-hypermucoviscosity- $\alpha$ hemolysis- $B s s S-f i m H$ & $0(0 \%)$ & $1(2 \%)$ \\
\hline $\begin{array}{l}\text { Biofilm-serum } \\
\text { resistant-haemagglutination-hypermucoviscosity-BssS-fimH-iss-iucA }\end{array}$ & $0(0 \%)$ & $1(2 \%)$ \\
\hline $\begin{array}{l}\text { Biofilm-serum } \\
\text { resistant-haemagglutination-hypermucoviscosity-lipase- } \alpha \text {-hemolysis-BssS-fimH-iss }\end{array}$ & $0(0 \%)$ & $1(2 \%)$ \\
\hline Biofilm-haemagglutination-hypermucoviscosity-gelatinase-BssS-fimH & $0(0 \%)$ & $1(2 \%)$ \\
\hline Biofilm-serum resistant-haemagglutination-hypermucoviscosity- $B s s S$-fim $H$-iss & $0(0 \%)$ & $2(4 \%)$ \\
\hline Biofilm-haemagglutination-hypermucoviscosity-lipase-BssS-fimH-iss-iucA & $0(0 \%)$ & $1(2 \%)$ \\
\hline Biofilm-serum resistant-haemagglutination-hypermucoviscosity-lipase- $B s s S$-fim $H$ & $0(0 \%)$ & $2(4 \%)$ \\
\hline $\begin{array}{l}\text { Biofilm-serum } \\
\text { resistant-haemagglutination-hypermucoviscosity-lipase-BssS-fimH-iss }\end{array}$ & $0(0 \%)$ & $1(2 \%)$ \\
\hline Total & $50(100 \%)$ & $50(100 \%)$ \\
\hline
\end{tabular}

\section{References}

[1] M. M. Gharrah, A. Mostafa El-Mahdy, and R. F. Barwa, "Association between virulence factors and extended spectrum betalactamase producing klebsiella pneumoniae compared to nonproducing isolates," Interdisciplinary Perspectives on Infectious Diseases, vol. 2017, Article ID 7279830, 14 pages, 2017. 


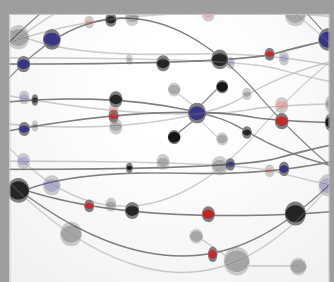

The Scientific World Journal
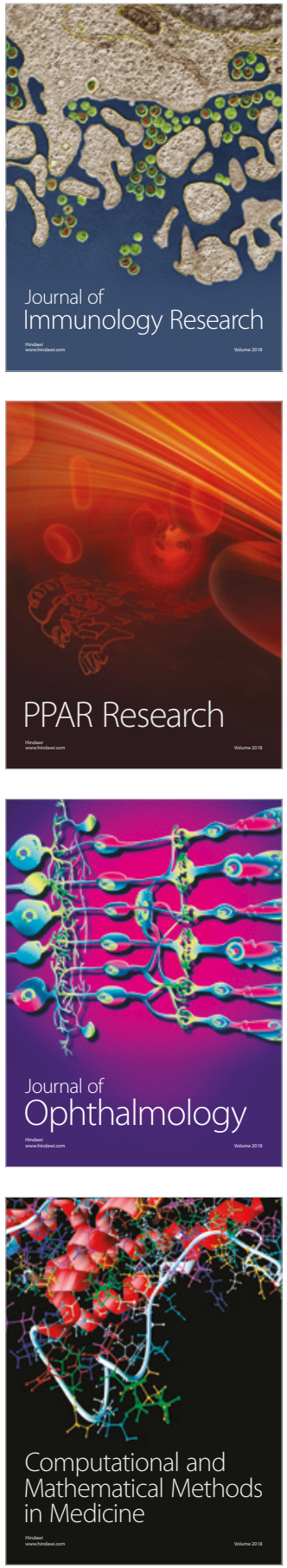

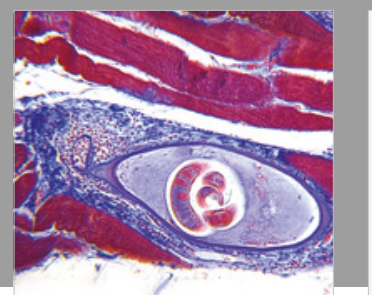

Gastroenterology Research and Practice

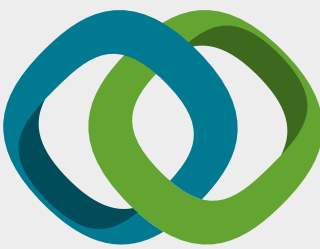

\section{Hindawi}

Submit your manuscripts at

www.hindawi.com
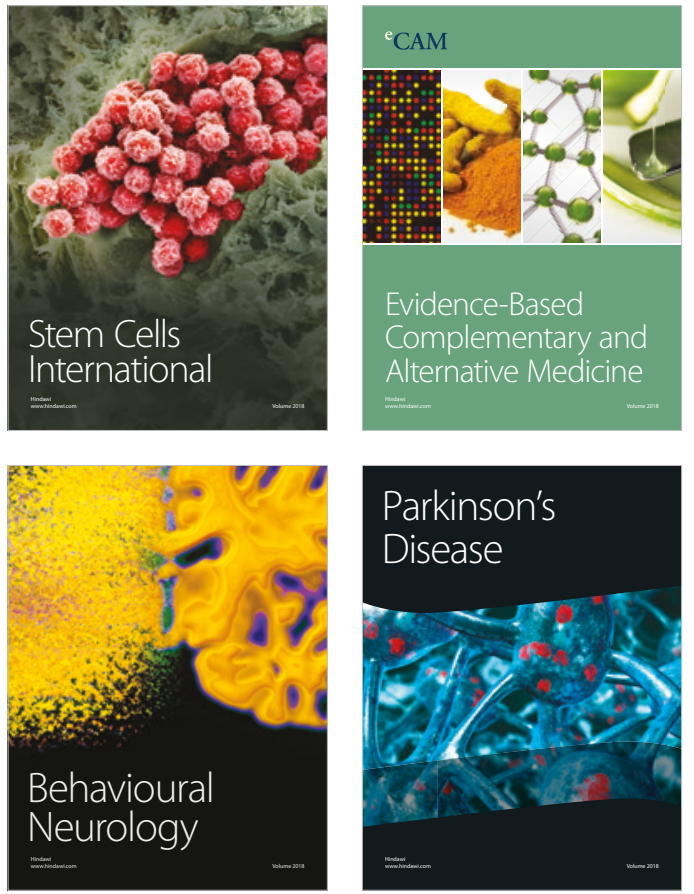

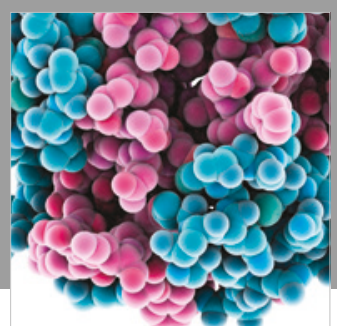

ournal of

Diabetes Research

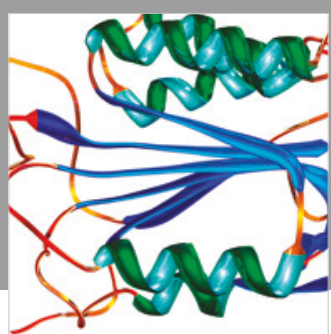

Disease Markers
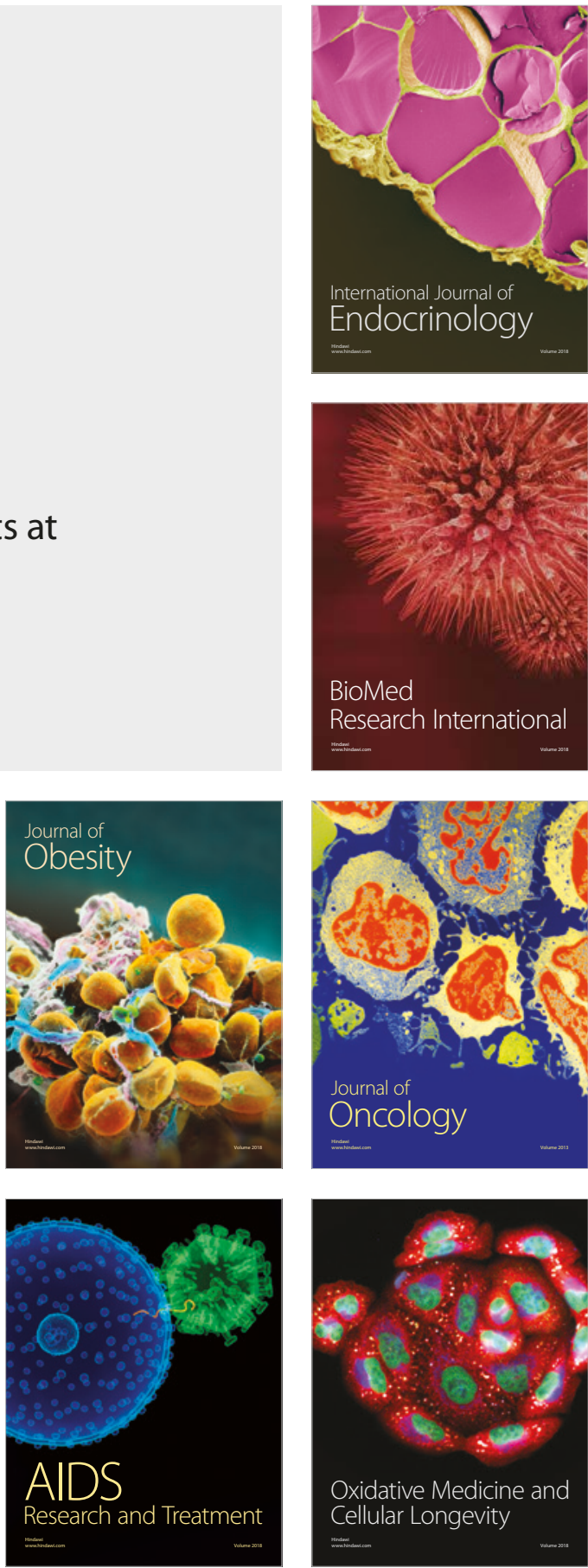